Solar Physics

DOI: $10.1007 / \bullet \bullet \bullet \bullet \bullet-\bullet \bullet \bullet-\bullet \bullet \bullet-\bullet \bullet \bullet \bullet-\bullet$

\title{
Solar Grand Minima and Random Fluctuations in Dynamo Parameters
}

\author{
D. Moss ${ }^{1} \cdot$ D. Sokoloff ${ }^{2}$ - I. Usoskin ${ }^{3}$. \\ V. Tutubalin ${ }^{4}$ \\ (C) Springer $\bullet \bullet \bullet$
}

\begin{abstract}
We consider to what extent the long-term dynamics of cyclic solar activity in the form of Grand Minima can be associated with random fluctuations of the parameters governing the solar dynamo. We consider fluctuations of the alpha-coefficient in the conventional Parker migratory dynamo, and also in slightly more sophisticated dynamo models, and demonstrate that they can mimic the gross features of the phenomenon of the occurrence of Grand Minima over a suitable parameter range. The temporal distribution of these Grand Minima appears chaotic, with a more or less exponential waiting time distribution, typical of Poisson processes. In contrast however, the available reconstruction of Grand Minima statistics based on cosmogenic isotope data demonstrates substantial deviations from this exponential law. We were unable to reproduce the non-Poissonic tail of the waiting time distribution either in the framework of a simple alpha-quenched Parker model, or in its straightforward generalization, nor in simple models with feedback on the differential rotation. We suggest that the disagreement may only be apparent and is plausibly related to the limited observational data, and that the observations and results of numerical modeling can be consistent and represent physically similar dynamo regimes.
\end{abstract}

Keywords: magnetic fields - Sun: magnetic fields - Sun: activity - Stars: magnetic fields - Stars: late-type

\section{Introduction}

The solar cycle is believed to be a result of dynamo action occurring somewhere inside the solar convective envelope. According to the classical Parker (1955)

\footnotetext{
${ }^{1}$ School of Mathematics, University of Manchester, Manchester M13 9PL, UK. email: moss@ma.man.ac.uk

2 Department of Physics, Moscow State University, Moscow, 119992, Russia. email: sokoloff@dds.srcc.msu.su

${ }^{3}$ Sodankylä Geophysical Observatory (Oulu Unit), 90014

University of Oulu, Finland. email: Ilya.Usoskin@oulu.fi

${ }^{4}$ Department of Mechanics and Mathematics, Moscow State

University, Moscow, 119992, Russia. email:

vntutubalin@yandex.ru
} 
model, this dynamo action can be envisaged as follows. Differential rotation $\Omega$ produces toroidal magnetic field from poloidal, while the " $\alpha$-effect" associated with the helicity of the velocity field produces poloidal magnetic field from toroidal. According to this scheme, the solar cycle length is identified with the dynamo time-scale, which can be estimated from the product of the amplitudes of the $\alpha$-effect and rotational shear $(\partial \Omega / \partial r, r$ being the radial coordinate), appropriately normalized with the turbulent diffusion coefficient (these yielding the dimensionless dynamo number), and with the turbulent diffusion time. The Parker model results in a periodic process in the form of propagation of a toroidal field pattern in the latitudinal direction (the "butterfly diagram"). A suitable choice of governing dynamo parameters gives equatorward pattern propagation as well as allowing the cycle period to agree with observations. More realistic dynamo models try to demonstrate that some plausible choice of parameters is compatible with available observational information from, say, helioseismology, or elaborates this simple scheme by various additional details, such as meridional circulation (see Petrovay, 2000; Charbonneau, 2005, for reviews).

In fact, the solar cycle is far from being a strictly periodic phenomenon. The amplitude of solar cycles varies substantially in time and reaches unusually large levels during the so-called Grand Maxima, one of which is now believed to be occurring. From time to time the level of solar cyclic activity becomes extremely low if not disappearing completely. Such minima of the cyclic activity are known as Grand Minima, the most well-known example being the Maunder Minimum, which occurred in the middle 17-th - beginning of 18-th centuries. The statistics of Grand Minima (and Maxima) can be to some extent reconstructed from data on cosmogenic isotope ${ }^{14} \mathrm{C}$ in tree rings (Usoskin et al., 2007). Quantification of the sequence of such events is still a contentious topic. It is important that the isotopic data provides a much longer record of Grand Minima/Maxima than do the sunspot observations. Moreover, the sequence of Grand Minima (and/or Maxima) appears to be random, rather than a periodic process.

It is known that simple deterministic numerical dynamo models of the solar cycle, which essentially develop the ideas of the Parker migratory dynamo, can give events comparable with Grand Minima/Maxima (e.g. Brandenburg et al., 1989), even showing behaviour which is irregular and chaotic in time (see e.g. Jennings and Weiss, 1991; Jennings, 1991; Tobias et al., 1995; Covas et al., 1998 - see also Moss and Brooke, 2000 in a more complex model). The presence of a long-term dynamics needs however an explanation. The most straightforward idea here is to recognize that the $\alpha$-effect, being the result of the electromotive force averaged over turbulent vortices, can contain a fluctuating contribution (Hoyng, 1993; Hoyng et al., 1994; Ossendrijver and Hoyng, 1996). The idea can lead to events similar to the Maunder Minimum on the timescale of centuries (see e.g. Tworkowski et al., 1998; also Brandenburg and Spiegel, 2008).

The aim of this paper is to investigate the long-term dynamics of solar activity by confronting the predictions of a Parker migratory dynamo model containing a random contribution to the $\alpha$-coefficient with the available data concerning the sequence of Grand Minima and Maxima, as inferred from the isotopic data. We also consider, more briefly, a more sophisticated dynamo model. Our general conclusion is that the fluctuations in the dynamo governing parameters can lead 
to phenomena similar to the Grand Maxima and Minima, in that the temporal distribution of the events appears chaotic.

We recognize a disagreement between the observational data and numerical simulations of our dynamo model, in that the statistics of the waiting time distributions of Grand Minima appear to have exponential tails, in contrast to the isotopic data in which the temporal distribution of Grand Minima and Maxima demonstrate a substantial deviation from exponential statistics. We argue that the disagreement plausibly is only apparent, and is connected with the limited extent in time of the observations, and that observations and modeling may represent physically similar dynamo regimes.

In this paper we concentrate on the distribution of Grand Minima.

\section{Long-term Dynamics of Solar Activity from the Isotopic Data}

Our intention is to test whether a simple physical model can reproduce the basic phenomena of the long-term solar dynamics. Of course, a detailed explanation of the phenomena needs a much more realistic model, including at least a 2D description of the solar magnetic field, realistic solar rotation curve, etc. Moreover, we do not exclude a priori that the phenomena could have some alternative explanation. We base our initial analysis on a simple illustrative model, rather than on something more realistic, in order to isolate physical phenomena and to take into account the quite limited status of the actual observational information.

We focus our attention on the long-term dynamics of solar activity on the time-scale of centuries. We note however that the idea of random fluctuations of the dynamo governing parameters can be instructive in explaining the stochastic features of short-term dynamics of solar activity, on the timescale of a few solar cycles (e.g. Moss et al. 1992; Hoyng et al., 1994).

We summarize the properties of the long-term solar dynamics based on the analysis of the data on cosmogenic isotope ${ }^{14} \mathrm{C}$ in tree-rings performed by Usoskin et al. (2007) for the Holocene (the last 11,000 years or about 1,000 solar cycles). From time to time, the cyclic solar activity as deduced from this proxy data demonstrates phenomena resembling Grand Maxima and Grand Minima. Usoskin et al. (2007) identified 27 Grand Minima of total duration of 1880 years (or about $17 \%$ of the entire period) during the 1,000 solar cycles ${ }^{1}$.

The sequence of Grand Maxima/Minima appears chaotic ${ }^{2}$. An important parameter characterizing intrinsic features of quasi-chaotic/stochastic processes is the distribution of waiting times (hereafter WTD) between successive Grand Minima. A simple Poisson process (i.e. with the probability of occurrence of Grand Minimum being constant and independent of the previous history of the

${ }^{1}$ Even though this data set is a reconstruction and may contain uncertainties, we will regard and refer to it as the "real" solar activity series throughout the paper, to emphasize its difference from the purely synthetic data modeled here.

${ }^{2}$ We do not claim here that this sequence $i s$ random in any mathematically rigorous sense, as proof of such a statement would require many more proxy Grand Minima/Maxima events than are available. 


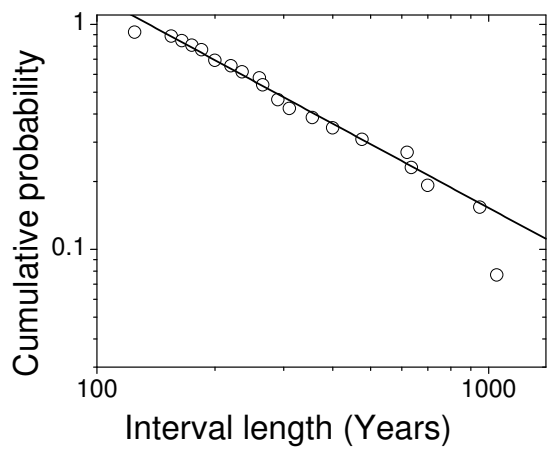

Figure 1. Waiting time distribution between the subsequent Grand Minima (see Usoskin et al., 2007) together with the best fit power law. The first and the last point were excluded from the fitting.

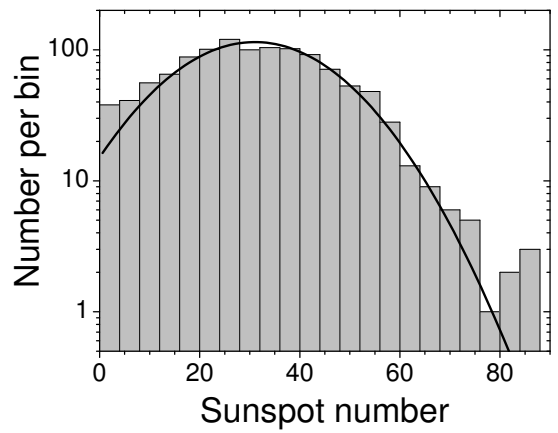

Figure 2. Histogram (the distribution function) of the decadal solar activity (quantified in sunspot number) during the Holocene (see Usoskin et al., 2007) together with the best fit normal distribution.

system) is characterized by an exponential WTD. On the other hand, significant deviation from an exponential tail in the WTD (e.g., such as a power law) implies an essentially non-Poisson process (e.g. self-organized criticality, or accumulation and release of energy). The WTD for solar Grand Minima identified via cosmogenic isotopes by Usoskin et al. (2007) displays significant deviation from exponential form (see Figure 2).

In the forthcoming sections we try to reproduce, in a statistical sense, the appearance of Grand Minima in the framework of different dynamo models. As criteria we will consider the shape of the distribution of waiting times between subsequent Grand Minima (exponential or power law) and the distribution function (DF) of the solar activity (assumed to correlate with sunspot number (SN) - see Figure 2.) 


\section{The Dynamo Model}

\subsection{The Parker Dynamo Models}

We first consider a simple dynamo model, a straightforward generalization of the initial Parker (1955) migratory dynamo. Following Parker, we average the meanfield equation with respect to the radial coordinate $r$ to arrive at the following set of equations (see e.g. Baliunas et al., 2006)

$$
\begin{gathered}
\frac{\partial B}{\partial t}=D g \sin \theta \frac{\partial A}{\partial \theta}+\frac{\partial^{2} B}{\partial \theta^{2}}-\mu^{2} B \\
\frac{\partial A}{\partial t}=\alpha B+\frac{\partial^{2} A}{\partial \theta^{2}}-\mu^{2} A
\end{gathered}
$$

A formal derivation of the above equations and detailed description of the nondimensionalization is given in, e.g., Sokoloff et al. (1996). Briefly, we use units of radius $R$ and global diffusion time $R^{2} / \eta$, where $\eta$ is the magnetic diffusivity. Here $B(\theta)$ represents the toroidal magnetic field and $A(\theta)$ is the azimuthal component of the vector potential for the poloidal field. $\theta$ is co-latitude (so $\theta=0$ corresponds to the north pole). $g=g(\theta)$ is the radial shear of the differential rotation. We cannot include a representation of a realistic solar rotation curve in our simple 1D model, and choose for the sake of simplicity $g=1$. $D$ is the dynamo number, which incorporates the intensity of both sources of generation, the alpha-effect and differential rotation, so the equations are given in nondimensional form. The terms with second derivatives are responsible for the latitudinal diffusion of magnetic field. The relaxation term proportional to $\mu^{2}$ represents the radial diffusion of magnetic field (see for details e.g. Kuzanyan and Sokoloff, 1996). We choose $\mu=3$, which corresponds to a convective shell occupying $1 / 3$ of the solar radius. Equantions (1) and (2) are solved by timestepping on a finite difference grid with $N$ points distributed uniformly in $0 \leq \theta \leq \pi$; our standard resolution is $N=101$.

We consider a simple algebraic quenching of $\alpha$-effect in the form

$$
\alpha=\frac{\alpha_{0}}{1+B^{2} / B_{0}^{2}},
$$

where $\alpha_{0}$ is a nominal, unquenched value of the alpha-effect and $B_{0}$ is a field strength at which dynamo action is stabilized by nonlinear effects, say the equipartition strength.

We appreciate that the actual form of dynamo quenching for solar dynamo can be much more sophisticated that the simple illustrative form (3) (cf. e.g. Gruzinov and Diamond, 1994). In particular, arguments of magnetic helicity conservation lead to a scheme with a differential equation for $\alpha$ (Zhang et al., 2006) which seems to give additional options to produce stochastic behavior of the dynamo generated magnetic field. Here, however, we restrict ourself to the simple scheme (3). 
We include $\alpha$-quenching by the toroidal field only, taking into account that the toroidal field is usually much larger than the poloidal. We keep the standard latitudinal profile $\alpha_{0} \propto \cos \theta$ and impose fluctuations which preserve the latitudinal profile in a given hemisphere by writing

$$
\alpha=\frac{\cos \theta}{1+B^{2}}\left(1+r_{i}(t)\right) .
$$

Thus we measure magnetic field in units of $B_{0}$ and incorporate the typical amplitude of the alpha-effect in the definition of dynamo number. The $r_{i}$ are pseudo-random numbers, supplied by a NAG Library routine; $i=1$ for the Northern (N) hemisphere, $i=2$ for the Southern (S). These values can be chosen independently, so we can model deviations in equatorial antisymmetry of the alpha-effect due to fluctuations. The time dependence of $r_{i}(t)$ is taken as a piece-wise constant function. The correlation time $T$ is chosen to be of order the nominal 11-year period. The fluctuations are supposed to be independent over different time ranges of length $T$, and Gaussian with standard deviation $\sigma$. We exclude very strong fluctuations, larger then $5 \sigma$. This basic model we refer to as Model I.

Our choice for the noise means that we consider global fluctuations of $\alpha$ on the temporal scales of order the cycle length and spatial (latitudinal) scale of the whole solar hemisphere. We do not include in our analysis variations of short time and latitudinal extent, on the scales of turbulent vortices, which are obviously important for the short-term dynamics of solar activity. The presence of longterm variations in the alpha-coefficient has been reported from analysis of direct numerical simulations by Brandenburg and Sokoloff (2002) and OtmianowskaMazur et al. (2006).

We also considered more sophisticated models for the noise.

(i) Model II. We now introduce in the sequences of time intervals fluctuations of $\alpha$ between intervals of random length in which there are no fluctuations. We define $\mu_{\Delta T}, \sigma_{\Delta T}$ to be the mean and standard deviations of the time intervals when there are zero perturbations to $\alpha_{N}, \alpha_{S}$. These parameters are the same for $\mathrm{N}$ and $\mathrm{S}$ hemispheres. Perturbations to $\alpha$ are $o$ for fixed time $t_{c}$ (as the previous model). Off times are of duration $\delta_{N, S}$, where $\delta_{N, S}$ are gaussian random variables with mean $\mu_{\Delta T}$ and standard deviation $\sigma_{\Delta T}$.

Limits are imposed on $\delta_{N, S}$ :

(a) if $\delta_{N, S}<0.20 \mu_{\Delta T}$, then $\delta_{N, S}=0.20 \mu_{\Delta T}$, to avoid negative/very close to zero intervals - important if $\mu_{\Delta T}$ and $\sigma_{\Delta T}$ are of similar size. So the minimum off interval is $0.2 \mu_{\Delta T}$.

(b) if $\delta_{N, S}>\mu_{\Delta T}+3 \sigma_{\Delta T}$, then $\delta_{N, S}=\mu_{\Delta T}+3 \sigma_{\Delta T}$.

Quite arbitrarily, we take $\sigma_{\Delta T} \sim \mu_{\Delta T}$.

(ii) Model III. We introduce a memory in the sequence of fluctuation of $\alpha$ as follows. Let $p_{n}$ be a sequence of independent pseudo-random gaussian numbers as supplied by the library routine (see above also). Then we introduce $r_{1}=$ $p_{1}, r_{2}=p_{2}$ and

$$
r_{n}=\frac{p_{n}+r_{n-1}}{\sqrt{2}} .
$$


The sequence $r_{n}$ has zero mean and the same standard deviation as $p_{n}$ (the factor $\sqrt{2}$ gives the normalization required). The memory time for the sequence $r_{n}$ is of order $T$.

(iii) Model IV. Now we implement a 3-level memory, by writing

$$
p_{n}=\frac{p_{n-2}+r_{n-1}+r_{n}}{\sqrt{3}} .
$$

We note in advance that these memory effects turn out not to play a crucial role in our analysis and discussion.

\section{2. $2 \mathrm{D}$ models with feedback on the differential rotation}

With the aim of exploring other possibilities, we also studied the behaviour of a 2D model in which the nonlinearity is the feedback of the Lorentz force on the differential rotation, using the code of Moss and Brooke (2000). In order to retain some of the spirit of the Parker dynamo, we considered a relatively thin shell, $0.8 \leq r \leq 1.0$ where $r$ is the fractional radius.

For Model $\mathrm{V}$, the initial rotation law is $\Omega \propto r$. We kept $N=101$ latitudinal grid points as for Models I-IV, but used only 11 uniformly distributed radial points, in what might be termed a $1.5 \mathrm{D}$ model. (Taking the inner radius of the shell at $r=0.9$ made no significant difference to our results.) The random perturbations were implemented as in Model I, with independent hemispheres. The standard dynamo parameters $C_{\alpha}, C_{\omega}$ were chosen to give a modestly supercritical dynamo, with unperturbed magnetic period of approximately 0.022 . We allowed the magnetic Prandtl number to take values of 1.0 and 0.01 . Unperturbed solutions were singly periodic.

For Model VI the initial rotation law has $\Omega \propto \sin ^{2} \theta$, in an attempt to capture the essence of the weak radial dependence of the solar rotation in the bulk of the upper half of the convection zone. $C_{\alpha}=5, C_{\omega}=-10^{4}$, otherwise Model VI was the same as Model V. One significant feature of Model VI is that the dynamo is steady near to marginal excitation. This can be explained by rotation laws with $\Omega=\Omega(\theta)$ being known to give radial migration of field patterns. However, here the restricted radial extent of the model inhibits this migration, resulting in a steady field. Notwithstanding this rather unsatisfactory feature, we present some results, as potentially illustrative of processes in the upper part of the solar CZ.

\section{Results}

We performed a number of numerical experiments. In most cases a modified Parker dynamo, as described above - i.e. as Models I-IV above - was used with $D=-10^{3}, \mu=3$ (a run in which $D=-3 \times 10^{3}$ is indicated by an asterisk in Table 4). Then the dimensionless period of the field $B$ (i.e. the nominal " $22 \mathrm{yr}$ " solar cycle period) is $\tau \approx 0.25$.

For all cases we performed "short" computations, over a dimensionless time 2500 (i.e. approximately 10000 cycles, which is an order of magnitude longer 
than the solar activity reconstructed over the Holocene). For a number of cases we made longer runs, over intervals of length 25000. We summarize the results obtained with Models I-IV in Table 1.

We give in Figure 4 an example of a run where we were unable to identify clearly Grand Minima/Maxima ( $\mathrm{a}$ - time series for $\alpha$, b - time series for toroidal field at an arbitrary point, $\mathrm{c}$ - histogram for the cycle intensity), while Figure 4 shows an example of a run with pronounced events resembling Grand Minima/Maxima.

For each model we consider $E$, the energy in the toroidal magnetic field. First, the series are averaged over the nominal undisturbed cycle length (the analogue of the 11-year cycle) and passed through the Gleissberg (12221) filter ${ }^{3}$, which is often applied when studying long-term variations of solar activity in order to suppress the noise (e.g., Gleissberg, 1944; Soon et al., 1996), similarly to the original analysis of Usoskin et al. (2007). Next, the distribution function of these averaged $E$ values was constructed and compared with that for sunspot activity (Figure 2). Here we are interested mostly in the probability of occurrence of low values, and thus the robustness of the definition of Grand Minima. Then we defined Grand Minima as periods when the cycle-averaged value of $E$ is systematically (for at least two consecutive cycles) below a threshold energy $E_{\text {th }}$. As discussed by Usoskin et al. (2007), Grand Minima of the solar activity are well-defined, due to the fact that the distribution function (Figure 2) is almost flat for SN below 10, implying an excess over a normal distribution. The DFs of the model runs were visually compared to the "real" one and placed into one of the five categories (see column 5 in Table 1): "OK", if the DF is similar to that of the solar SN; "+", if there is a clear excess of low values (i.e the simulated activity is dominated by the Grand Minima, and thus the statistics of their occurrence cannot be clearly determined) - "++" denotes a stronger excess; and "-" that the low values are underrepresented compared to that of the solar record. For some parameters the DF is bimodal and this is indicated in the corresponding column of Table 1. We note that the identification of Grand Minima is relatively robust for the DF similar to the "real" one (denoted as "OK") in the Table, whereas it depends on the choice of $E_{\text {th }}$ for both "+" and "." DFs. In our model, Grand Minima are not a special state of the dynamo, but rather the result of random fluctuations, and thus they cannot be unambiguously identified.

Next, the cumulative probability $\mathrm{WTD}^{4}$ was constructed, in a similar way to that shown in Figure 2, and the shape of its tail was assessed as being either close to an exponential, or deviating from it. This is indicated in column 6 of the Tables. If a significant deviation from an exponential tail was observed, the same model was rerun with new randomization and a 10-times longer realization in order to improve the statistics. In all such cases the WTD returns to a nearly perfect exponential form when the enhanced statistics are taken into account. In other cases (notably Model VI) a non-exponential tail may appear for a limited

\footnotetext{
${ }^{3}$ The 12221 filtering is defined as $\left\langle x_{i}\right\rangle=0.125\left(x_{i-2}+2 x_{i-1}+2 x_{i}+2 x_{i+1}+x_{i+2}\right)$.

${ }^{4}$ The waiting time is defined between centres of successive Grand Minima.
} 


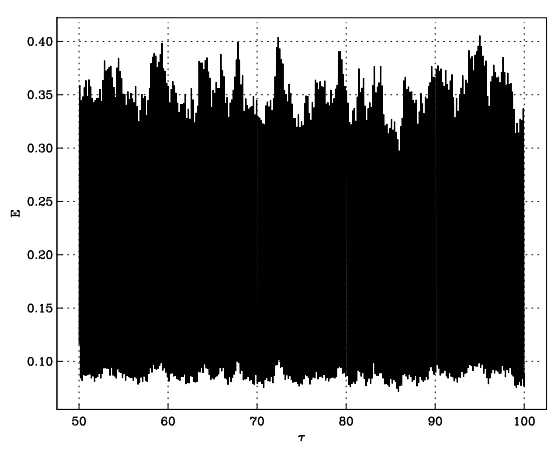

Figure 3. Time series of total energy for Model I, with $T=0.03, \sigma=0.05$. This case does not show well defined Grand Minima-like events.

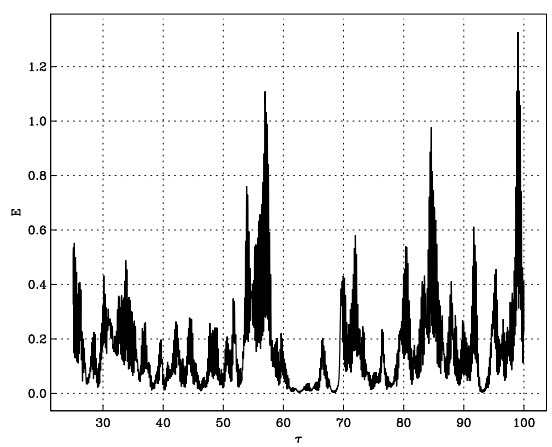

Figure 4. Time series of total energy for Model III, with $T=0.15, \sigma=0.12$. Here Grand Minima-like events can be identified.

range of threshold values $E_{\mathrm{th}}$, but disappears for larger and smaller values. All such cases (deviation from exponential WTD tail with normal statistics that disappears with enhanced statistics or fragility with respect to choice of $E_{\mathrm{th}}$ ) are denoted by "exp" in Tables 1 and 2. We stress that the entries in the WTD column refer to the tail of the distribution of the waiting time between successive Grand Minima, whereas the DF entries refer to the shape of the histogram of SN ( $E$ values) at low activity level.

The general result of this investigation is that the simple model under consideration reproduces in a suitable parameter range many features of the sequence of Grand Minima, as deduced from the available observations. It means that we can in a more or less clear way separate the epoch of normal cycles and Grand Minima, and that the sequence of Grand Minima looks random rather than periodic. We note again that the model considered does not contain any specific mechanism to produce Grand Minima, rather that the Grand Minima occur as a result of random fluctuations of the parameters governing the dynamo. We appreciate that the model does not reproduce the available phenomenology completely, i.e. we were unable to reproduce the non-Poissonic tails of the WTD of the isotopic record. 
Table 1. Results for selected cases of Models I-IV. Model parameters are: $n$ - run number (an asterisked run number run means that $D=-3 \times 10^{3}$ instead of usual $D=-10^{3}$ ), $\iota=1$ means that the $\alpha$-fluctuation is the same in both hemisphere while $\iota=2$ means that the fluctuations in both hemisphere are independent, $\sigma$ is standard deviation and $T$ the mean of the random "off" times (for models I, II, IV the "off" times are constant, and so $T$ is this constant value). Columns under "Results": DF gives the nature of the Distribution function (excess/lack of low values denoted as "+" /"-", "++" denotes a very strong excess, bimodal stands for bimodal DF); WTD describes the shape of WTD ("exp" stands for the clear exponential tail, while "exp*" denotes cases when a deviation from the exponent is observed in some parameter range with lower statistucs but becomes exponential with higher statistics.) In run $38^{\dagger}$ we also tried a different long realization of random fluctuations with the same governing parameters as in the run 38 . See the text for further details.

\begin{tabular}{|c|c|c|c|c|c|}
\hline \multicolumn{4}{|c|}{ Model parameters } & \multicolumn{2}{|c|}{ Results } \\
\hline$n$ & $\iota$ & $\sigma$ & $T$ & $\mathrm{DF}$ & WTD \\
\hline \multicolumn{6}{|c|}{ Model I } \\
\hline 2 & 1 & 0.2 & 0.12 & - & $\exp$ \\
\hline 3 & 1 & 0.4 & 0.12 & $\mathrm{OK}$ & $\exp$ \\
\hline 4 & 1 & 0.6 & 0.12 & OK & $\exp$ \\
\hline 5 & 2 & 0.2 & 0.12 & OK & $\exp$ \\
\hline 6 & 2 & 0.4 & 0.12 & + & $\exp$ \\
\hline 7 & 2 & 0.2 & 0.06 & - & $\exp$ \\
\hline $8^{*}$ & 2 & 0.2 & 0.20 & bimodal & exp* \\
\hline 9 & 2 & 0.2 & 0.12 & bimodal & $\exp$ \\
\hline 10 & 2 & 0.3 & 0.12 & + & $\exp$ \\
\hline 11 & 2 & 0.25 & 0.12 & OK & exp* \\
\hline 12 & 2 & 0.25 & 0.06 & - & exp* \\
\hline 13 & 2 & 0.25 & 0.24 & + & $\exp$ \\
\hline \multicolumn{6}{|c|}{ Model II } \\
\hline 21 & 2 & 0.25 & 0.12 & + & $\exp$ \\
\hline 22 & 2 & 0.30 & 0.12 & + & $\exp$ \\
\hline \multicolumn{6}{|c|}{ Model III } \\
\hline 31 & 2 & 0.25 & 0.12 & + & $\exp$ \\
\hline 32 & 2 & 0.25 & 0.20 & ++ & $\exp$ \\
\hline 33 & 2 & 0.25 & 0.06 & ++ & $\exp$ \\
\hline 34 & 2 & 0.12 & 0.06 & - & $\exp$ \\
\hline 35 & 2 & 0.12 & 0.20 & + & $\exp$ \\
\hline 36 & 2 & 0.15 & 0.12 & OK & exp* \\
\hline 37 & 2 & 0.15 & 0.06 & OK & exp* \\
\hline 38 & 2 & 0.15 & 0.20 & OK & exp* \\
\hline $38^{\dagger}$ & 2 & 0.15 & 0.20 & OK & exp* \\
\hline \multicolumn{6}{|c|}{ Model IV } \\
\hline 41 & 2 & 0.15 & 0.12 & OK & exp* \\
\hline 42 & 2 & 0.10 & 0.12 & - & $\exp$ \\
\hline 43 & 2 & 0.15 & 0.20 & OK & exp* \\
\hline
\end{tabular}


Solar Grand Minima and random fluctuations in dynamo parameters

Table 2. Results for selected cases for Models IV and VI. Notation is as for Table 1, except that Pr, the magnetic Prandtl number, is also tabulated; the label "exp*" is explained in the text.

\begin{tabular}{ccccccc}
\hline \multicolumn{4}{c}{ Model parameters } & \multicolumn{2}{c}{ Results } \\
\hline$n$ & $i$ & $\sigma$ & $T$ & $P r$ & DF & WTD \\
\hline \multicolumn{6}{c}{ Model V } \\
\hline 61 & 2 & 0.2 & 0.05 & 1.0 & - & $\exp$ \\
62 & 2 & 0.4 & 0.005 & 1.0 & OK & $\exp$ \\
63 & 2 & 0.6 & 0.005 & 0.01 & OK & $\exp$ \\
\hline \multicolumn{1}{c}{ Model VI } \\
\hline 71 & 2 & 0.20 & 0.005 & 1.0 & - & $\exp$ \\
72 & 2 & 0.20 & 0.005 & 0.01 & - & $\exp$ \\
73 & 2 & 0.20 & 0.010 & 1.0 & - & $\exp$ \\
74 & 2 & 0.40 & 0.010 & 1.0 & - & $\exp$ \\
75 & 2 & 0.40 & 0.010 & 0.01 & ++ & $\exp *$ \\
76 & 2 & 0.20 & 0.010 & 0.01 & - & $\exp$ \\
\hline
\end{tabular}

We have also investigated to what extent the results are robust when a more complicated semi-2D model is considered. Table 2 gives results for Models $\mathrm{V}$ and VI - see also the discussion in Sect 6 . Of course, there are many possible ways to make the model more realistic and it is practically impossible to search the parameter space in full detail. However, at least at first sight, the general message seems to be clear: for the more complicated models we do not see anything basically new compared with the simpler one.

\section{Long-term Dynamics of Solar Activity}

Let us summarize the results obtained. We have tried to simulate the occurrence of solar Grand Minima, as being an effect of fluctuations in the governing parameters in a simple model of the solar dynamo, i.e. the Parker migratory dynamo. The model demonstrates some aspects of this phenomenon within a suitable range of the dynamo parameters. The sequence of simulated Grand Minima appears chaotic and in this sense is similar to the observational data. The statistics of Grand Minima occurrence looks however different from that reconstructed from observations. While the WTD of observed Minima demonstrates a substantial deviation from the exponential tail, the WTD of the simulated results is nearly Poissonic.

We see that the effect of fluctuations is quite robust in the sense that we do not obtain non-exponential tails in the probability distribution of waiting times between Grand Minima/Maxima in any of our simulations. Of course, the details of the sequence of Grand Minima/Maxima are model dependent. We even tried 
to modify the idea under discussion quite substantially by replacing the alphaquenching nonlinearity by feedback of the Lorentz force on the rotation law. In all these cases we still obtained Poisson-like distributions of Grand Maxima/Minima (or no such events at all). However, our investigations are largely limited to 1D models (i.e. models which can be considered as a direct generalization of the original Parker scheme), except for some experiments with the Lorentz force nonlinearity, where a nominal radial dependence was introduced in what might be termed a "1.5D" model.

Further elaboration of the dynamo model to fully 2D form necessitates substantially more complicated numerics, and our ability to clarify the situation in detail is limited. We did demonstrate however by one example that even fully 2D models with Lorentz force feedback and on an underlying solar-like rotation law give a Poisson-like sequence of Grand Minima/Maxima. On the other hand, there still remain some hopes that more complicated dynamo models (e.g. Moss and Brooke, 2000; Brooke et al., 2002) can provide examples of determinent intermittency, i.e. highly non-Poissonic behaviour of the Grand Minima/Maxima sequence, for certain parameter choices, notably a small value of the magnetic Prandtl number.

We believe that the disagreement between statistics of model results and observations is plausibly related to the limited length of the observational record, which is not long enough to be considered as statistically stationary in time. On the other hand, the time series of numerical models can be made long enough to be statistically stationary. When the length of a simulated data series is comparable with the length of observational data, a significant deviation from the exponential WTD, similar to the observations, is found in several simulations. However, this deviation disappears in all analyzed runs after extension of the simulated series (such cases are denoted as "exp*" in Tables 1 and 2.

These differences can be related to the limited length of the series which is not enough to reach statistical stationarity, as suggested by the following test. Let us consider a time series $f_{n}=f\left(t_{n}\right)$ where the instants of observations $t_{i}=n \tau$. If the length of the time series is sufficient to consider it as a stationary random process then the random quantity $g_{n}=\Sigma_{0}^{n} f_{i}$ is expected to behave as $g_{n}=\langle f\rangle n+h(n)$ where $\langle f\rangle$ is the mean of the random process $f_{n}$ and $h(n)$ is a function that grows more slowly then $n$. By plotting $g_{n}$ against $n$ and comparing the results with a linear trend we can check whether the time series is long enough to be consider as a realization of a stationary random process. We performed this test with the record of solar activity which has been exploited to provide the sequence of Grand Minima (Figure 5a here) and recognize there substantial deviations from linear-law behaviour. We compare it with the simulated time series for certain of the runs indicated as "exp*". We show in Figure 5b the results obtained for the entire run 41 (of length 15873) - it appears close to linear. If however we restrict the length of this simulated series to the first 4000 records the cumulative function demonstrates substantial deviations from a linear behavior (Figure 5c). Other runs marked as "exp*" in the Tables 1 and 2 demonstrate similar results when this test is applied.

Note that the discussion above assumes implicitly that solutions of the dynamo models with fluctuations in the dynamo governing parameters can contain 

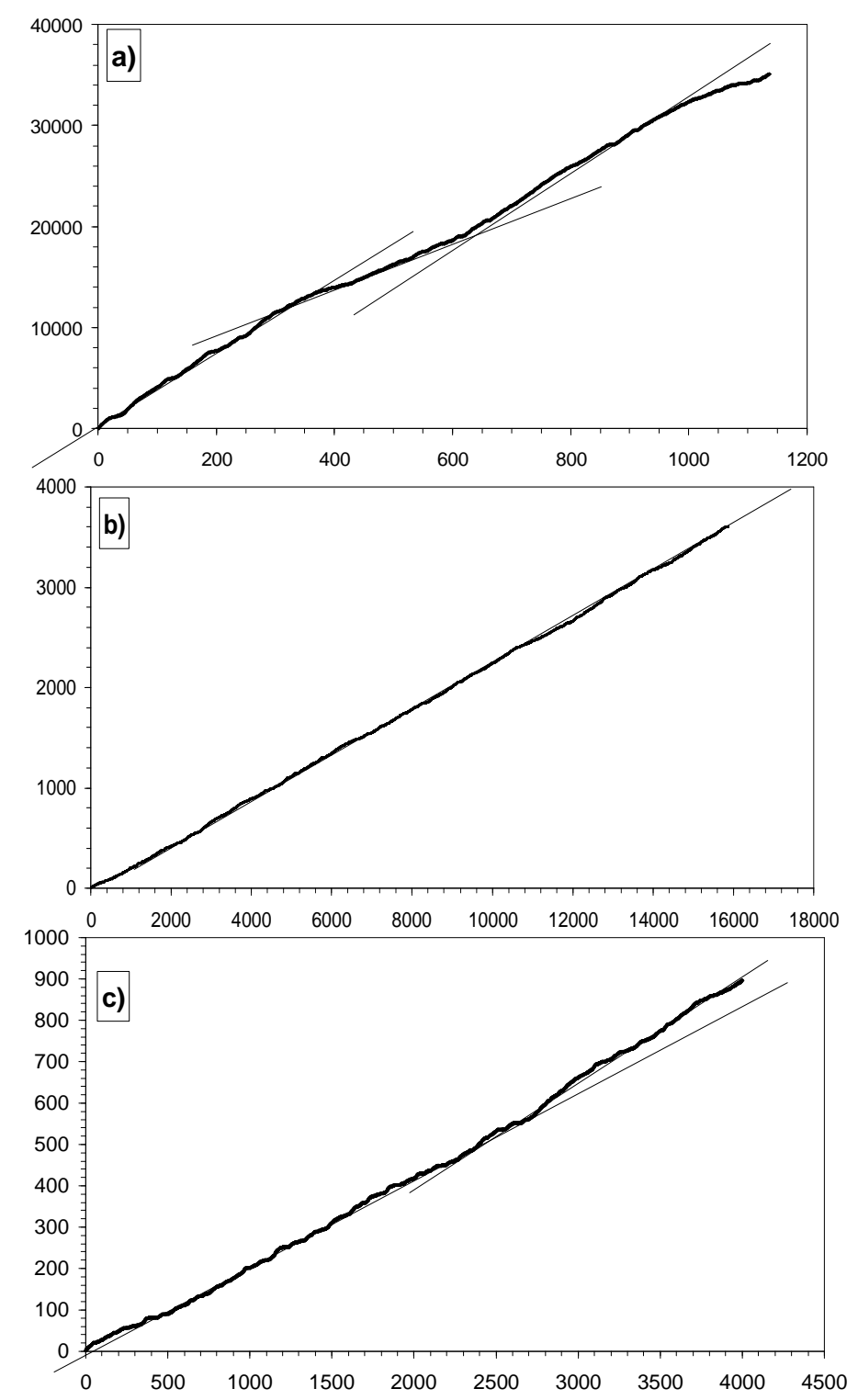

Figure 5. (a) Cumulative sunspot number against time, (b) run 41, full dataset, cumulative energy vs time, (c) run 41, truncated dataset, cumulative energy vs time; we show strait lines which approximates the plots presented 
time scales much longer than the cycle length and the average time between Grand Minima. Justification of such assumptions needs specific numeric simulations which are outside of the scope of this investigation.

We conclude that the observational data demonstrate similar behaviour to the data with simulated data of the runs indicated as "exp*" provided the comparable timescales are considered. We expect that the observations of solar activity would give exponential waiting time distribution for the sequence of Grand minima provided such long record would be available.

\section{Discussion and Conclusions}

We have demonstrated that the phenomenon of the occurrence of solar Grand Minima can be simulated as an effect of fluctuations in the governing parameters in a simple model of solar dynamo at least in the framework of the interpretation of observation suggested. We stress that the limited nature of the observations available does not make it possible to compare the results of simulations and observations in complete detail ; however we do not see in the observational data anything that is basically incompatible with the simulations. Thus, simulations in regimes marked as "exp*" in the tables look close to the observed phenomenology and might be regarded to be not inconsistent with the observations.

However, since the results cannot be directly compared in the statistical sense, other possibilities which exist to explain the seeming disagreement between simulations and observations deserve consideration. First of all, the phenomena of Grand Minima and Maxima may be associated with fine details of the solar dynamo (for example, the exact shape of the solar rotation law), rather than being a general property of nonlinear dynamos in a spherical shell for a suitable parameter range. (Our experiments with a realistic rotation law in a 2D dynamo model, although not encouraging, were too limited to rule this out completely.) A further possibility is that the dynamo mechanism itself produces a Poissonlike sequence of Grand Maxima/Minima, but there are also long-term trends in solar hydrodynamics (on the scale of thousand years) which affect the timescale of the weighting time and mimics the non-Poisson behaviour. This could be, for example, via the Reynolds stresses that drive the differential rotation. Another option is that the non-Poissonic nature of the observed sequence of Grand Minima/Maxima is an artefact of the limited statistics.

We stress once more that we present here a development of a very strong suggestion that the solar dynamo engine does not contain any specific mechanism that produces Grand Minima (and Maxima), but that they are rather a result of random fluctuations in the dynamo governing parameters. On one hand, the ability to convert random noise into a sequence of clearly separated events looks an intriguing feature of the dynamo. This ability however can be considered as an example of intermittency, which is a known property of various nonlinear systems where it can produce various spatial or/and temporal structures from random noise (see e.g. Zeldovich et al., 1991). On the other hand, it looks more than plausible that the solar dynamo does possess something specific that allows fine 
tuning of WTD of Grand Minima, which produces non-Poisson tails. However we can not identify this feature of the dynamo engine at the moment, and realize that the physical mechanism behind the occurrence of Grand Minima may be only partly or not at all related to random fluctuations (e.g. Petrovay, 2007).

We appreciate that the problem considered here belongs to the general topic of the influence of noise, which is addressed in many fundamental papers. Our ability to exploit the deep methods suggested in this area (e.g. Abarbanel et al., 1993 ) is however restricted by the limited nature of the available observational data. Note however that we have incorporated some memory effects into models III, IV and V so that, in principle at least, we are going slightly beyond studying the effects of random noise and the expected associated chaos. Also that it is not a priori altogether obvious how these random inputs will appear after passing through the dynamo "machine".

Our work is based on the tacit assumption that the sunspot number is linearly related to the magnetic energy in the dynamo. This is a common asssumption in solar cycle modelling, but it is quite possible that the number and size of the active regions appearing on the surface might be more plausibly taken to be proportional to the toroidal flux, rather than to the energy. However this option leads to similar conclusions to those presented above. On the other hand, it also seems possible that there could be a threshold effect at play here, so that active regions only emerge if the toroidal field strength exceeds some minimal value (e.g. Ruzmaikin, 2001). This would introduce a marked nonlinearity into the relationship between activity indices and toroidal field parameters. It is clear that the results obtained can be sensitive to this nonlinearity which, in principle, we could investigate. Accordingly, our investigation of the Grand Minima phenomenon has to be considered to some extent as illustrative until the influence of the nonlinearity is resolved. A further possibility in a $2 \mathrm{D}$ model is to use the toroidal flux or energy in the immediate sub-surface region as a proxy for surface activity.

Finally, we mention the regime with so-called "dynamo outbursts" observed in the VKS dynamo experiment (Ravelet et al., private communication) as presenting one further topic that may be relevant to our investigation. We note that episodes from the time series of magnetic field evolution taken from a sensor in this experiment look very similar to that presented in Figure 4.

We recognize that our results are not positive in the sense of answering in a clear-cut manner the key question of whether a simple model, such as we have considered, is able to reproduce the observed statistics of the occurrence of solar Grand Minima. However we do feel that we may have provided some insight into the question of the statistical stability of the observations, and to have provided information and guidance for future investigations.

Acknowledgements D.S. is grateful to the Royal Society for supporting his visit to Manchester, and also to RFBR for financial support under grant 07-02-00127. D.M. and D.S. thank the Finnish Academy of Science and Letters (Väisälä foundation) for supporting their visits to the University of Oulu.

\section{References}

Abarbanel, H.D., Brown, R., Sidorowich, J.J., Tsimring, L.Sh.: 1993, Rev. Mod. Phys. 651331. 
Baliunas S., Frick P., Moss D., Popova E., Sokoloff D., Soon W.: 2006, Mon. Not. R. Astron. Soc. 365181

Brandenburg, A, Sokoloff D.: 2002, Geophys. Astrophs. Fluid Dyn. 96319.

Brandenburg, A, Spiegel, E.: 2008, arXiv: 0801.2156 - submitted to Astron. Nachr.

Brandenburg, A., Krause F., Meinel R., Tuominen I., Moss D.: 1989, Astron. Astrophys. 213, 411.

Brooke, J. Moss, D. Phillips, A.: 2002, Astron. Astrophys. 395, 1013.

Charbonneau, P.: 2005, Living Reviews in Solar Physics 2, No. 2 (http://solarphysics.livingreviews.org/Articles/lrsp-2005-2/)

Covas, E., Tavakol, R., Tworkowski, A., Brandenburg, A.: 1998, Astron. Astrophys. 329, 350.

Gleissberg, W.: 1944, Terr. Magnet. Atmosph. Electr. 49, 243.

Gruzinov, A.V., Diamond, P.H.: 1994, Phys. Rev. Lett. 72, 1651.

Jennings, R.L.: 1991, Geophys. Astrophys. Fluid Dyn. 57, 147.

Jennings, R.L., Weiss, N.O.: 1991, Mon. Not. R. Astron. Soc. 252, 249.

Hoyng, P: 1993, Astron. Astrophys. 272, 321.

Hoyng, P., Duistermaat, J. J.: 2004, Europhys. Lett. 68, 177.

Hoyng P., Ossendrijver M. A. J. H., Schmitt D.: 2001, Geophys. Astrophys. Fluid Dyn. 94, 263.

Hoyng, P., Schmitt, D., Teuben, L.J.W.: 1994, Astron. Astrophys. 289, 265.

Kuzanyan, K.M., Sokoloff, D.D.: 1996, Astron. Rep. 40, 425.

Moss, D., Brandenburg, A., Tavakol, R.K., Tuominen I.: 1992, Astron. Astrophys. 265, 843.

Moss, D., Brooke, J.: 2000, Mon. Not. R. Astron. Soc., 315, 521.

Otmianowska-Mazur, K., Kowal, G., Hanasz, M.: 2006, Astron. Astrophys. 445, 915.

Ossendrijver, A.J.H., Hoyng, P.: 1996, Astron. Astrophys. 313, 959.

Parker, E.: 1955, Astrophys. J. 122, 293.

Petrovay, K., 2000, in: Wilson, A. (ed.) The solar cycle and terrestrial climate, Solar and space weather, ESA SP-463, 3.

Petrovay, K.: 2007, Astton. Nachr. 328, 777.

Schmitt, D., Ossendrijver, M. A. J. H., Hoyng, P.: 2001, Geophys. Astrophys. Fluid Dyn. 94, 283.

Sokoloff, D.D., Fioc, M., Nesme-Ribes, E.: 1996, Magnetohydrodynamics 31, 18.

Soon, W.H., Posmentier, E.S., Baliunas, S.L.: 1996, Astrophys. J. , 472, 891.

Stefani, F., Xu, M., Sorriso-Valvo, L., Gerbeth, G., Günther, U.: 2007, Geophys. Astrophys. Fluid Dyn. 101, 227.

Tobias, S.M., Weiss, N.O., Kirk, V.: 1995, Mon. Not. R. Astron. Soc. , 273, 1150.

Tworkowski, A., Tavakol, R., Brandenburg, A., Brooke, J.M., Moss, D., Tuominen, I.: 1998, Mon. Not. R. Astron. Soc., 296, 287.

Usoskin, I.G., Solanki, S.K., Kovaltsov, G.A: 2007, Astron. Astrophys. 471, 301.

Zeldovich, Ya.B., Ruzmaikin, A.A., Sokoloff, D.D: 1991, The Almighty Chance, World Science, Singapore.

Zhang, H., Sokoloff, D., Rogachevskii, I., Moss, D., Lamburt, V. Kuzanyan, K., Kleeorin, N.: 2006, Mon. Not. R. Astron. Soc., 365, 276. 